\title{
Stability-Constrained Aerodynamic Shape Optimization of a Flying Wing Configuration
}

\author{
Charles A. Mader* \\ University of Toronto Institute for Aerospace Studies \\ Toronto, Ontario, Canada \\ Joaquim R.R.A. Martins ${ }^{\dagger}$ \\ Department of Aerospace Engineering, University of Michigan \\ Ann Arbor, Michigan
}

\begin{abstract}
Stability-constrained aerodynamic shape optimization is conducted using three dimensional, time-spectral computational fluid dynamics. To facilitate this, a method for computing stability constraints with the time-spectral method is introduced. The unique characteristics of this method allow an adjoint technique to be used to generate the gradients of the stability constraints that are required for optimization. These gradients are generated using the automatic differentiation adjoint technique, which provides accurate and efficient results. The resulting stability constraints and their gradients are used in the optimization of a flying wing.
\end{abstract}

\section{Introduction}

Aerodynamic optimization is a rapidly maturing field. Using adjoint methods with Euler and NavierStokes computational fluid dynamics (CFD), a number of researchers have successfully solved drag minimization problems. ${ }^{1-5}$ However, when considering unconventional designs - such as a flying wing with no distinct horizontal tail - there is a strong coupling between the aerodynamic efficiency of the aircraft and its stability characteristics. This coupling leads to a different optimal solution from a stability-constrained optimization than that which is obtained from a simple aerodynamic optimization.

In this work we conduct aerodynamic optimization with basic, static longitudinal stability constraints included. Two approaches are considered. In the first approach, we constrain $C_{m}$ to be approximately zero and $C_{M_{\alpha}}$ to be negative about the center of gravity $(\mathrm{CG})$ of the aircraft. This combination of parameters ensures that the aerodynamic moments caused by perturbations from the cruise condition are restoring moments, giving a statically stable aircraft. In the second approach, we constrain the value $C_{m_{\alpha}}+C_{L_{\alpha}} \times$ Static Margin to be zero. This allows the optimization to return a design with a predefined static margin at the optimal solution.

Unlike the force coefficients $\left(C_{L}, C_{D}\right.$ and $\left.C_{m}\right)$, which can be computed directly with any flow solver, stability derivatives, such as $C_{m_{\alpha}}$ and $C_{m_{q}}$, are themselves derivative quantities and require a special flow solver for their computation. In addition, in order to be able to conduct gradient based optimization, these modifications must be made in a way that allows the gradients of these quantities to be computed in an efficient manner. In this work, the stability derivative, $C_{m_{\alpha}}$, is calculated using a new method based on a time-spectral CFD solution. The algebraic method used in conjunction with the time-spectral solution allows the use of an adjoint method for the derivative computation, which achieves the desired efficiency. The details of this method are provided in Section III

*Ph.D Candidate, AIAA Student Member

†AssociateProfessor, AIAA Senior Member

Copyright (C) 2010 by the authors. Published by the American Institute of Aeronautics and Astronautics, Inc. with permission 
Due to the relatively high computational cost of the CFD analysis and the potentially large number of design variables involved in shape optimization, a gradient-based method is selected for optimization. In this case we use an SQP algorithm $\left(\mathrm{SNOPT}^{6}\right)$ and an automatic differentiation adjoint method (ADjoint ${ }^{7}$ ) to compute the gradients required by the optimizer. These tools are used to demonstrate the effect of static longitudinal stability constraints on an aerodynamic optimization problem. Optimizations have been conducted on cases with and without the stability constraints and the resulting designs are compared to highlight the effects of the stability and trim constraints on the optimum design.

\section{Time-Spectral Method}

The underlying CFD algorithm used in this work is a time-spectral Euler solver. The basics of the method are repoduced here. For a more detailed explanation the reader is referred to the original works by van der Weide et al. ${ }^{8}$ and Gopinath and Jameson. ${ }^{9}$

We start by defining the governing equations for unsteady flow. These can be written as:

$$
V \frac{\partial w}{\partial t}+\frac{\partial f_{i}}{\partial x_{i}}=0
$$

where $x_{i}$ are the coordinates in the $i^{\text {th }}$ direction. For the Euler equations, the state and the fluxes for each cell are:

$$
w=\left[\begin{array}{c}
\rho \\
\rho u_{1} \\
\rho u_{2} \\
\rho u_{3} \\
\rho E
\end{array}\right], \quad f_{i}=\left[\begin{array}{c}
\rho u_{i} \\
\rho u_{i} u_{1}+p \delta_{i 1} \\
\rho u_{i} u_{2}+p \delta_{i 2} \\
\rho u_{i} u_{3}+p \delta_{i 3} \\
\rho u_{i} H
\end{array}\right] .
$$

To simplify notation, we will rewrite this equation as:

$$
V \frac{\partial w}{\partial t}+R(w)=0
$$

To formulate the time-spectral equations, we assume that the flow is periodic in time and express the solution of the states, $w$, as a Fourier series and its inverse transform:

$$
\begin{aligned}
\hat{w}_{k} & =\frac{1}{N} \sum_{n=0}^{N-1} w^{n} e^{i k \frac{2 \pi}{T} n \Delta t}, \\
w^{n} & =\sum_{k=-\frac{N}{2}}^{\frac{N}{2}-1} \hat{w}_{k} e^{i k \frac{2 \pi}{T} n \Delta t},
\end{aligned}
$$

where $N$ is the number of time intervals, $T$ is the period of the solution and $\Delta t=T / N$. We can then evaluate the partial derivative of the states with respect to time as:

$$
D_{t} w^{n}=\frac{2 \pi}{T} \sum_{k=-\frac{N}{2}}^{\frac{N}{2}-1} i k \hat{w}_{k} e^{i k \frac{2 \pi}{T} n \Delta t}
$$

which can be combined with Equation (4) to form a matrix operator,

$$
D_{t} w^{n}=\sum_{j=0}^{N-1} j_{n}^{j} w^{j}
$$

which connects all of the time instances in the solution. Thus, we end up solving the equivalent of $N$ simultaneous steady state systems based on the equation:

$$
V D_{t} w^{n}+R\left(w^{n}\right)=0
$$

2 of 14 
where $R\left(w^{n}\right)$ are the steady-state residuals for the $n^{\text {th }}$ time instance.

For the work in this paper we use the SUmb flow solver ${ }^{10}$ that has been developed at Stanford University under the sponsorship of the Department of Energy. SUmb is a cell-centered multiblock solver for the Reynolds-averaged Navier-Stokes equations (steady, unsteady, and time-spectral) and it has options for a variety of turbulence models with one, two and four equations. In this work, we limit ourselves to the time-spectral Euler equations.

\section{Stability Derivatives}

A key development in this work is the addition of stability constraints to aerodynamic optimization. The computation of stability derivatives using CFD is not a new idea: several techniques have been demonstrated previously, including finite differences, ${ }^{11}$ automatic differentiation ${ }^{12,13}$ and analytic sensitivity methods. ${ }^{14}$ While these methods have proven to be effective in predicting stability derivatives, they each use well established first derivative methods to compute the stability derivatives. Including these quantities as constraints in an optimization problem requires the computation of their gradients. Since the quantities in question are themselves derivatives, the computation of their gradients would require second derivative information from the CFD solver. This is a computationally expensive proposition and for large numbers of design variables would be prohibitively expensive.

The time-spectral approach presented here does not have that limitation. To evaluate stability derivatives from a time-spectral solution, a series of linear regressions are performed on the periodic solution produced by the CFD solver. Because this approach is simple and algebraic, an adjoint technique can be used over the entire process to calculate the gradients for optimization. A similar method for computing the stability derivatives with a periodic steady solution has been demonstrated by Murman, ${ }^{15}$ who uses a frequency domain method to produce periodic data for relatively large amplitude oscillations ( \pm 2.5 deg.). This data can then be analyzed with the same techniques used to produce stability derivatives from forced oscillation wind tunnel data, which allows the method to take advantage of the large body of knowledge that has been developed in that field. In our case, we work with much smaller amplitude oscillations designed to isolate specific motion variables individually. This allows individual derivatives to be computed directly. The virtual nature of the CFD solutions allows the simulation of these motions, which would be very difficult to reproduce physically.

To develop this method, we refer to the section on linear air reactions in Etkin. ${ }^{16}$ From this theory we know that for a general motion, the force and moment coefficients of an aircraft — for example, the lift coefficient - can be approximated as:

$$
C_{L}=C_{L_{0}}+C_{L_{\alpha}} \alpha+C_{L_{\dot{\alpha}}} \dot{\alpha}+C_{L_{\ddot{\alpha}}} \ddot{\alpha}+\ldots+C_{L_{\beta}} \beta+\ldots,
$$

where all motion states $\alpha, \beta, V, p, q, r, Z_{E}$ and their associated time derivatives are included. However, if we simplify the motion to consist of a single dynamic state - for example $\alpha$ - the value of the remaining motion states are zero and they, and their associated derivatives, drop out of the equation. In the case of a pure $\alpha$ motion this leads to:

$$
C_{L}=C_{L_{0}}+C_{L_{\alpha}} \alpha+C_{L_{\dot{\alpha}}} \dot{\alpha}+C_{L_{\ddot{\alpha}}} \ddot{\alpha}+\ldots
$$

If we assume that the higher order derivatives are small and neglect them, we get:

$$
C_{L}=C_{L_{0}}+C_{L_{\alpha}} \alpha+C_{L_{\dot{\alpha}}} \dot{\alpha} .
$$

Diagrams illustrating pure $\alpha$ and $q$ motions are shown in Figures 1 and 2 respectively. In these diagrams, the grid is moving with respect to a stationary air mass and the arrows in the diagrams indicate the relative motion of the incoming flow with respect to the grid. The $\alpha$ motion, shown in Figure 1, is an oscillating vertical motion with the forward grid velocity modified in sync with the oscillations such that the incoming velocity maintains a constant magnitude, but changes direction. Time instances 2 and 3 are at the top and bottom of the oscillations, respectively, illustrating the points in the cycle where the grid experiences pure forward motion. Time instance 1 illustrates a point midway through the oscillation where the grid is experiencing both forward and vertical motion. The diagram in Figure 2 illustrates a pure $q$ motion. In this case, the grid rotates with an oscillating value of $q$ and the magnitude and direction of the velocity are modified such that the incoming $\alpha$ is constant. 


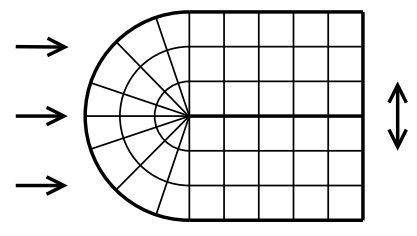

Time Interval 1

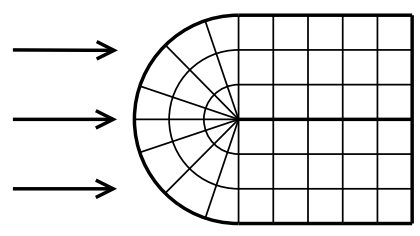

Time Interval 2

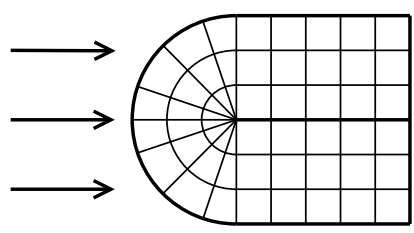

Time Interval 3

Figure 1. Pure angle of attack motion
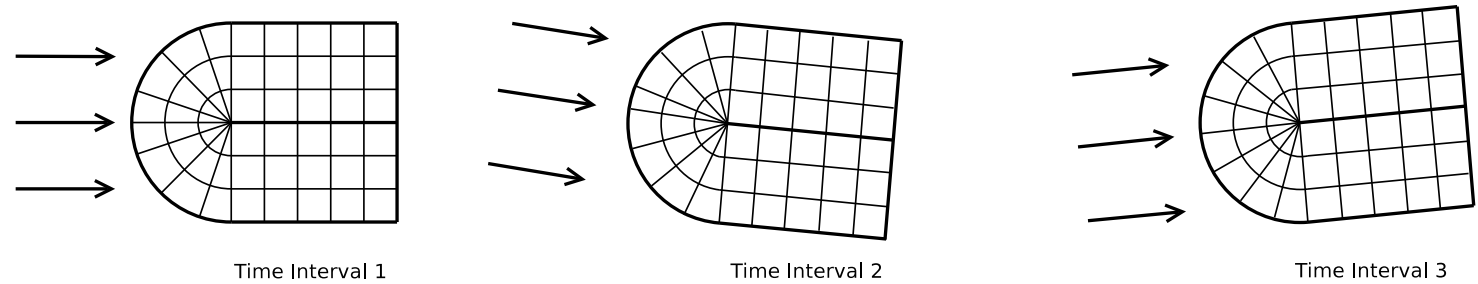

Figure 2. Pure pitching motion

When one of these pure motions is simulated with the time-spectral method, the result is a solution that consists of force coefficient $\left(C_{L}, C_{D}\right)$ and moment coefficient $\left(C_{m_{x}}, C_{m_{y}}, C_{m_{z}}\right)$ values at $N$ time instances in a periodic solution. A set of solutions corresponding to a pure $q$ motion are shown in Figure 3. To compute stability derivatives from this solution, we compute a linear least squares fit of the output coefficient (e.g., $C_{L}$ ) with respect to the primary motion variable from the solution (e.g., $q$ ). The slope of the line resulting from this fit is the stability derivative $\left(C_{L_{q}}\right)$ while the $y$-intercept of the line is the value of $C_{L}$ at $q=0$.

To validate this approach, derivatives for the NACA 0012 airfoil are computed using the time-spectral method and compared to the numeric results published by Limache et al. ${ }^{14}$ These reference results are based on computations from an inviscid two-dimensional CFD solver. The solver was modified to include a non-inertial reference frame formulation, allowing the dynamic stability derivatives to be computed from a steady solution. In the reference work, those derivatives were computed using an analytic, direct sensitivity method. The results of these comparisons are shown in Table 1 . Figure 3 corresponds to the Mach $=0.5$ case in Table 1 .

Table 1. Time-spectral stability derivatives for a NACA 0012 airfoil at $\alpha=0.0$ degrees

\begin{tabular}{lccc}
\hline \hline Derivative & Time-Spectral (3 Point) & Reference $^{14}$ & \% Difference \\
\hline \multicolumn{4}{c}{ Mach $=0.1$} \\
$C_{L_{q}}$ & 9.626 & 10.377 & $7.24 \%$ \\
$C_{M_{q}}$ & -3.349 & -3.489 & $4.02 \%$ \\
& Mach $=0.5$ & & \\
$C_{L_{q}}$ & 11.860 & 11.847 & $0.11 \%$ \\
$C_{M_{q}}$ & -4.002 & -3.968 & $0.86 \%$ \\
& Mach $=0.8$ & & \\
$C_{L_{q}}$ & 21.064 & 21.889 & $3.81 \%$ \\
$C_{M_{q}}$ & -7.928 & -8.884 & $10.7 \%$ \\
\hline \hline
\end{tabular}

As is demonstrated in the table above, the derivatives computed with the time spectral results agree well with the reference results: for the Mach $=0.5$ case the results match with a relative difference that is less 


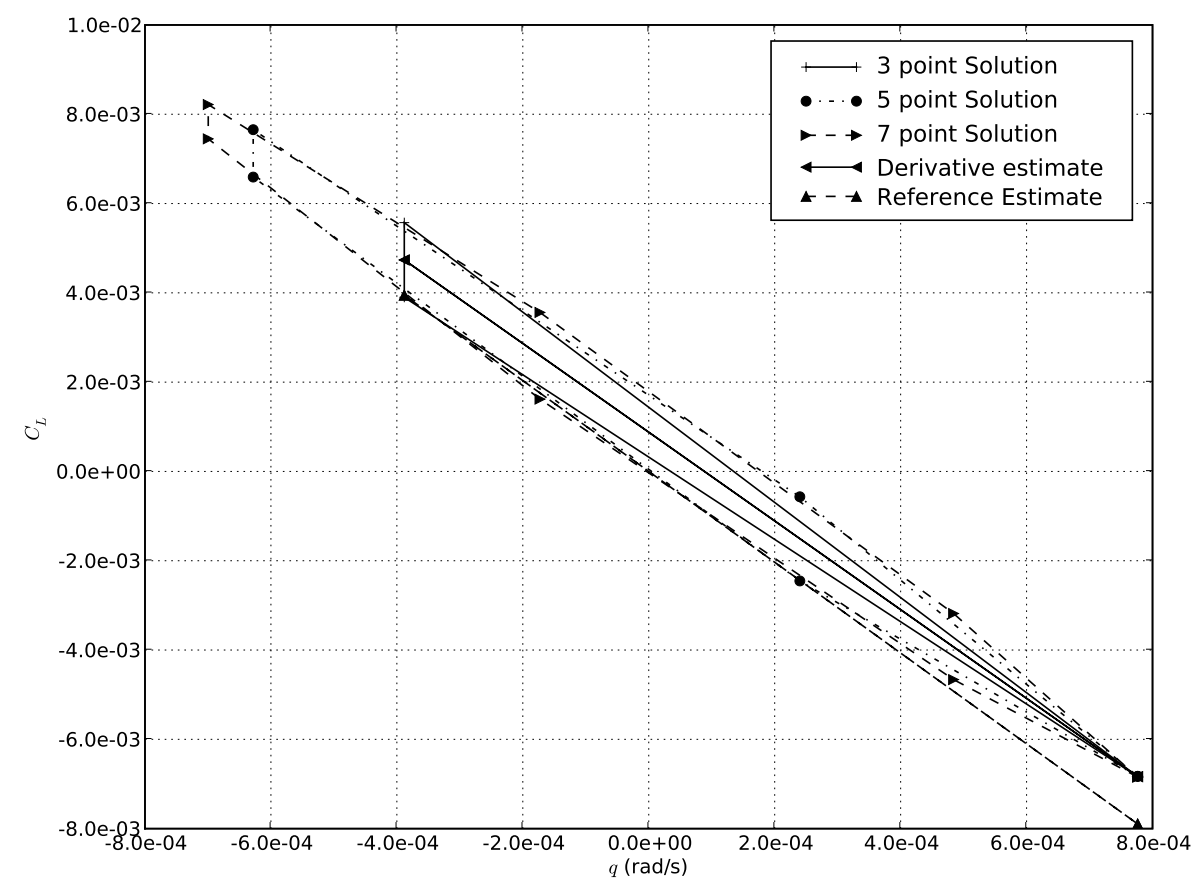

Figure 3. Time spectral $q$ derivative: Mach $=0.5$

than one percent. The maximum difference occurs for Mach $=0.8$, where $C_{m_{q}}$ differs by almost $11 \%$. A possible reason for the larger discrepancy in the Mach $=0.8$ case is the existence of a shock in the solution. A slight variation in the predicted location of the shock between the two solutions would cause a significant variation in the prediction of $C_{m}$ and any other values derived from it.

\section{Time-Spectral ADjoint Method}

The time-spectral derivatives are computed directly from the coefficient values calculated in the flow solution. Therefore, an adjoint approach applied to the solver is sufficient to compute the gradients of the coefficients that are required for the optimization. We start by writing the vector-valued function, $I$, that we want to differentiate as:

$$
I=I\left(x, w^{n}(x)\right),
$$

where $x$ represents the vector of design variables and $w^{n}$ is the state variable vector for the $n^{\text {th }}$ time instance, where $n=1, \ldots, N$. For a given vector $x$, the solution of the governing equations of the system yields a vector $w^{n}$, thus establishing the dependence of the state of the system on the design variables. In general, we denote these governing equations by:

$$
\mathcal{R}\left(x, w^{n}(x)\right)=0 .
$$

However, since the flow solution is time-spectral in nature, from van der Weide et al., ${ }^{8}$ we define:

$$
R_{T S}=V D_{t} w^{n}+R\left(w^{n}\right)=0,
$$

where $R\left(w^{n}\right)$ is a normal steady-state residual for the $n^{t h}$ time instance, where $n=1, \ldots, N$, and $D_{t}$ is a spectral operator that spans all $N$ time instances. Now we have a modified residual:

$$
\mathcal{R}_{\mathcal{T} \mathcal{S}}\left(x, w^{n}(x)\right)=0,
$$


which can be treated in the same fashion as the steady state residual would be in a normal adjoint formulation. We use the chain rule to write the total sensitivity of the vector-valued function $I$ as:

$$
\frac{\mathrm{d} I}{\mathrm{~d} x}=\frac{\partial I}{\partial x}+\frac{\partial I}{\partial w^{n}} \frac{\mathrm{d} w^{n}}{\mathrm{~d} x} .
$$

Further, because the governing equations must always be satisfied at a converged solution, the total derivative of the residuals 15 with respect to any design variable must also be zero. Expanding the total derivative of the governing equations with respect to the design variables we can write:

$$
\frac{\mathrm{d} \mathcal{R}_{\mathcal{T S}}}{\mathrm{d} x}=\frac{\partial \mathcal{R}_{\mathcal{T S}}}{\partial x}+\frac{\partial \mathcal{R}_{\mathcal{T} \mathcal{S}}}{\partial w^{n}} \frac{\mathrm{d} w^{n}}{\mathrm{~d} x}=0 .
$$

This expression provides the means of eliminating the total derivative $\mathrm{d} w^{n} / \mathrm{d} x$ from the total sensitivity computation for $I$. Moving the first term of this equation to the right hand side, we get:

$$
\frac{\partial \mathcal{R}_{\mathcal{T} \mathcal{S}}}{\partial w^{n}} \frac{\mathrm{d} w^{n}}{\mathrm{~d} x}=-\frac{\partial \mathcal{R}_{\mathcal{T S}}}{\partial x} .
$$

Substituting the solution of this system into Equation (16) yields:

$$
\frac{\mathrm{d} I}{\mathrm{~d} x}=\frac{\partial I}{\partial x}-\frac{\partial I}{\partial w^{n}}\left[\frac{\partial \mathcal{R}_{\mathcal{T} \mathcal{S}}}{\partial w^{n}}\right]^{-1} \frac{\partial \mathcal{R}_{\mathcal{T} \mathcal{S}}}{\partial x} .
$$

The adjoint approach consists in factorizing the $\partial \mathcal{R}_{\mathcal{T} \mathcal{S}} / \partial w^{n}$ matrix with the term to its left, yielding the adjoint system:

$$
\left[\frac{\partial R_{T S}}{\partial w}\right]^{T} \psi=\frac{\partial I}{\partial w} .
$$

Then, this solution is used in Equation (19) to obtain the total sensitivity:

$$
\frac{d I}{d x_{D V}}=\frac{\partial I}{\partial x_{D V}}-\psi^{T} \frac{R_{T S}}{\partial x_{D V}} .
$$

This yields a sensitivity method with a computational cost that is essentially independent of the number of design variables. More details on the ADjoint method can be found in Mader et al. ${ }^{7}$ Note that because the time-spectral system is $N$ times the size of the steady-state solutions, the adjoint system is also $N$ times larger than the equivalent steady-state system.

To improve the accuracy and reduce the implementation time of the adjoint solver, we use automatic differentiation to compute the values of the partial derivatives. More specifically, we use a reverse mode, source transformation tool (Tapenade ${ }^{17,18}$ ) to generate derivative code for each of the partial derivative terms. This reverse mode approach is very efficient for the objective function partial derivatives, as the number of objectives is far less than the number of flow states and design variables. The efficiency of the reverse mode approach is less apparent for the residual partial derivatives. However, as described in Mader et al. ${ }^{7}$ a modified, single cell version of the residual calculation is used, which makes the reverse mode computation extremely efficient for this calculation. To facilitate the solution of the large, sparse linear systems represented by these equations, we use the Portable, Extensible Toolkit for Scientific Computation (PETSc). ${ }^{19,20}$ This is a sparse linear and non-linear solution package that provides a variety of parallel solution and preconditioning algorithms.

\section{Center of Gravity Calculation}

Both the moment coefficient, $C_{m}$, and all of its derivatives - in this case $C_{m_{\alpha}}$ - are strongly dependent on the center of gravity ( $\mathrm{CG}$ ) location. In order to facilitate the optimizations described in Section $\mathrm{C}$, a simple, wing center of gravity calculation was implemented. The method used is derived from the work of Chai et al. ${ }^{21}$ They state that the wing center of gravity for a normal transport wing is located between the fore and aft spar along the wing mean aerodynamic chord (MAC). That idea is used as the basis for the method described below. We start by calculating the MAC and the location of its quarter chord. These calculations are based on the methods presented in ESDU item $76003 .{ }^{22}$ This provides a basis on which to 
locate the fore and aft spars for the wing. As part of the problem definition, the locations of the spars are defined. In this work we have assume that the forward spar is at $25 \%$ of the MAC and the aft spar is at $75 \%$ of the MAC. We can then specify the location of the CG as a percentage of the distance between the spars $\left(C G_{\% M A C}\right)$. This gives a reference location about which the moment coefficient, $C_{m}$, and all of its derivatives can be calculated.

\section{Results}

The results are presented in four sections. Section A describes the test case used and presents some basic solutions for the case. Section $\mathrm{B}$ presents the results relating to the performance and accuracy of the timespectral adjoint method used in this work, and Section C presents the results relating to the optimizations conducted on flying wings. Comparisons between the various optimization results are discussed in Section D

\section{A. Test Case}

The test case used in this work is the ONERA M6 wing, commonly used in CFD. The test case is run at Mach $=0.8$ with a symmetry condition at the root and inviscid wall boundary conditions. The solver used is SUmb, ${ }^{10}$ a three dimensional, structured, multi-block solver with a cell-centered, finite-volume scheme. The grid contains 155,648 cells and is shown in Figure 4(a). A steady state solution is shown in Figure 4(b) and a time-spectral solution is shown in Figure 5 .

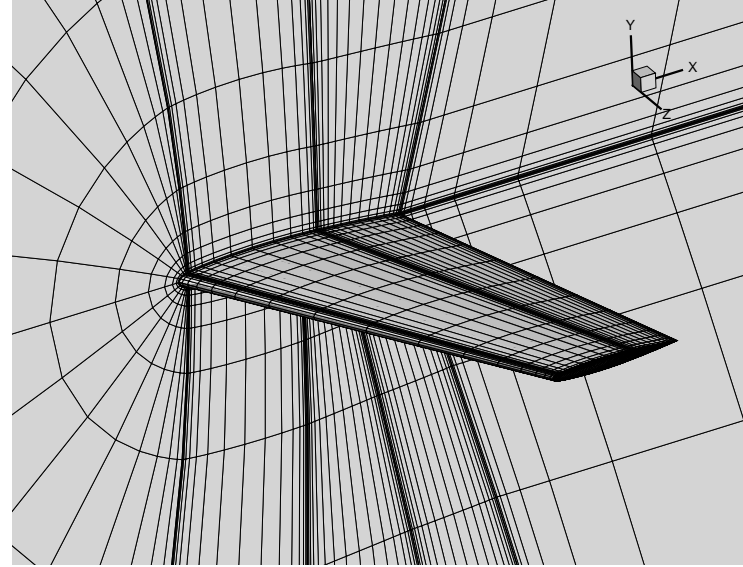

(a) ONERA M6: mesh

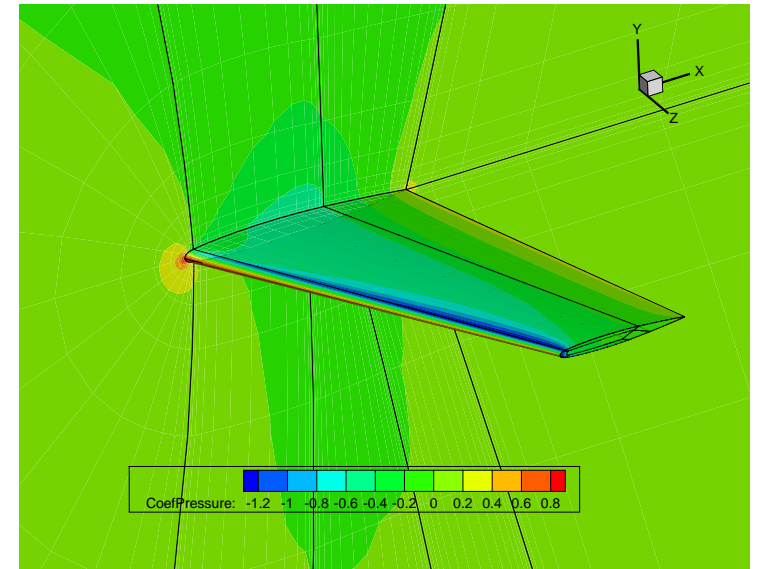

(b) ONERA M6: surface $C_{p}$ distribution: Mach $=0.8$ $\alpha=3.0 \mathrm{deg}$.

Figure 4. ONERA M6 test case

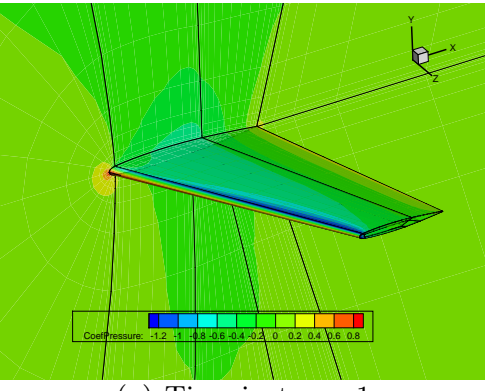

(a) Time instance 1

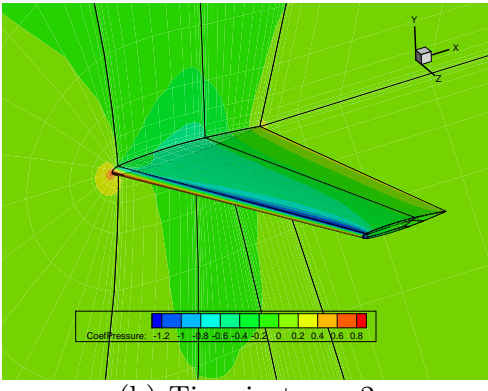

(b) Time instance 2

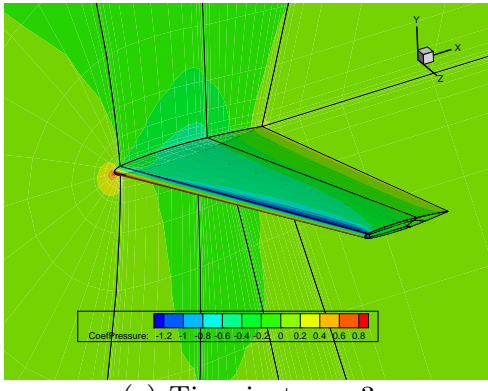

(c) Time instance 3

Figure 5. ONERA M6: time-spectral surface $C_{p}$ distribution, Mach=0.8, $\alpha=3.0 \mathrm{deg}$. 


\section{B. Performance}

To demonstrate the performance of the methods developed, accuracy and timing results are shown below.

Table 2. Sensitivity verification: Onera M6 test case 155,648 cells: $10^{-10}$ convergence

\begin{tabular}{llrr}
\hline \hline Cost Function & Design Variable & Adjoint & Complex Step \\
\hline$C_{L}$ & $\alpha$ & 4.7375673877687 & 4.737567386045973 \\
$C_{D}$ & $\alpha$ & $1.22477869862 \mathrm{E}-2$ & $1.224778698891853 \mathrm{E}-2$ \\
$C_{m}$ & $\alpha$ & $-7.47987636404091 \mathrm{E}-1$ & $-7.479876364269280 \mathrm{E}-1$ \\
$C_{m_{\alpha}}$ & $\alpha$ & -1.8465959882458 & -1.846596078281581 \\
\hline$C_{L}$ & Sweep & $-2.7252398443603 \mathrm{E}-5$ & $-2.725239835642640 \mathrm{E}-5$ \\
$C_{D}$ & Sweep & $-1.3086996 \mathbf{7 1 E}-4$ & $-1.308699669258835 \mathrm{E}-4$ \\
$C_{m}$ & Sweep & $1.2288071756500 \mathrm{E}-4$ & $1.228807175870053 \mathrm{E}-4$ \\
$C_{m_{\alpha}}$ & Sweep & $1.280213841445 \mathrm{E}-1$ & $1.280213841476047 \mathrm{E}-1$ \\
\hline$C_{L}$ & Twist & $2.8603177646382 \mathrm{E}-2$ & $2.860317764808717 \mathrm{E}-2$ \\
$C_{D}$ & Twist & $3.141227092 \mathrm{E}-4$ & $3.141227051424936 \mathrm{E}-4$ \\
$C_{m}$ & Twist & $-3.4019066938546 \mathrm{E}-3$ & $-3.401906695603677 \mathrm{E}-3$ \\
$C_{m_{\alpha}}$ & Twist & $-6.52457602472 \mathrm{E}-2$ & $-6.524575982519138 \mathrm{E}-2$ \\
\hline$C_{L}$ & Taper & $-3.3182460374741 \mathrm{E}-3$ & $-3.318246037774965 \mathrm{E}-3$ \\
$C_{D}$ & Taper & $-6.474634336 \mathrm{E}-4$ & $-6.474634317703330 \mathrm{E}-4$ \\
$C_{m}$ & Taper & $1.9513202619885 \mathrm{E}-5$ & $1.951320291763559 \mathrm{E}-5$ \\
$C_{m_{\alpha}}$ & Taper & $3.49418901607 \mathrm{E}-2$ & $3.494189001133485 \mathrm{E}-2$ \\
\hline \hline
\end{tabular}

The derivatives shown in Table 2 clearly demonstrate the accuracy of the derivative method used. In most cases the derivatives are accurate to at least 8 digits, with the worst case being a 6 digit disagreement and the best case 10 digit agreement. This is extremely good agreement, especially when considering that the solution is converged to a relative level of $10^{-10}$, which means that 10 digit agreement is the best that can be expected from an numerically exact derivative method.

\begin{tabular}{lrr}
\hline \hline & \multicolumn{2}{c}{ Onera M6 Case } \\
\hline Number of processors & Steady-State & Time-Spectral \\
Number of cells & 38 & 38 \\
Number of time instances & 155,648 & 155,648 \\
\hline Flow solution & 241.01 & 356.13 \\
\hline ADjoint & 102.19 & 380.47 \\
\hline Breakdown: & & \\
Computation of residual matrices & 24.21 & 109.24 \\
Computation of reconditioning matrix & 7.50 & 72.96 \\
Computation of volume to surface partial derivative & 2.00 & 5.63 \\
Computation of RHS & 0.29 & 0.70 \\
Solution of the adjoint equations & 67.13 & 191.34 \\
Computation of total sensitivities & 1.06 & 0.60 \\
\hline \hline
\end{tabular}

Table 3. ADjoint computational cost breakdown (times in seconds)

The timing results shown in Table 3 demonstrate the efficiency of the approach and implementation of the ADjoint method. Comparing the ADjoint solvers to the flow solvers, in both cases the ADjoint takes less than half as long as the flow solver (0.4 and 0.44 times respectively). Given that this is a reverse derivative that computes the derivative of each output with respect to all of the input values, this is even

$$
8 \text { of } 14
$$


more attractive for problems with large numbers of design variables. To illustrate, consider a simple timespectral optimization problem with 50 design variables and two output variables, one objective and one constraint. A finite difference method would require 50 additional flow solutions - 11.8 hours - for a single gradient evaluation, while the adjoint method would require 2 adjoint solutions - 13 minutes - for the same computation. The cost advantage of using the adjoint approach is evident. Further, the cost of using the time-spectral computation instead of a steady computation is also reasonable. The time-spectral flow solver is 3.5 times as costly as the steady solver and the time-spectral adjoint is 3.8 times as costly as the steady adjoint. While a factor of four increase in cost is significant, it is a reasonable cost to pay to be able to include stability characteristics in the optimization process.

\section{Optimization}

To assess the effects of stability constraints on aerodynamic shape optimization results, four optimization problems are examined. To serve as a baseline case, the following lift-constrained drag minimization problem is solved:

$$
\begin{aligned}
\text { minimize } & C_{D}\left(x, w^{n}(x)\right) \\
\text { w.r.t. } & x=[\alpha, \text { sweep, twist, taper }] \\
\text { subject to } & R\left(x, w^{n}(x)\right)=0 \\
& C_{L_{\text {target }}}-C_{L}\left(x, w^{n}(x)\right) \leq 0
\end{aligned}
$$

As an intermediate case, a trim constraint is added to the problem by limiting the moment coefficient,i.e., $0 \leq C_{m} \leq 10^{-4}$. This gives:

$$
\begin{aligned}
\text { minimize } & C_{D}\left(x, w^{n}(x)\right) \\
\text { w.r.t. } & x=\left[\alpha, \text { sweep, twist, taper, } x_{C G_{\text {target }}}, C G_{\% M A C}\right] \\
\text { subject to } & R\left(x, w^{n}(x)\right)=0 \\
& C_{L_{\text {target }}}-C_{L}\left(x, w^{n}(x)\right) \leq 0 \\
& 0 \leq C_{m}\left(x, w^{n}(x)\right) \leq 10^{-4} \\
& -10^{-5} \leq x_{C G}-x_{C G_{\text {target }}} \leq 10^{-5}
\end{aligned}
$$

For the first stability constrained problem, the $C_{m_{\alpha}}$ constraint is added, giving:

$$
\begin{aligned}
\text { minimize } & C_{D}\left(x, w^{n}(x)\right) \\
\text { w.r.t. } & x=\left[\alpha, \text { sweep, twist, taper, } x_{C G_{\text {target }}}, C G_{\% M A C}\right] \\
\text { subject to } & R\left(x, w^{n}(x)\right)=0 \\
& C_{L_{\text {target }}}-C_{L}\left(x, w^{n}(x)\right) \leq 0 \\
& 0 \leq C_{m}\left(x, w^{n}(x)\right) \leq 10^{-4} \\
& C_{m_{\alpha}}\left(x, w^{n}(x)\right) \leq 0 \\
& -10^{-5} \leq x_{C G}-x_{C G_{\text {target }}} \leq 10^{-5}
\end{aligned}
$$

For the second stability constrained problem, the static margin constraint replaces the $C_{m_{\alpha}}$ constraint, giving:

$$
\begin{aligned}
\text { minimize } & C_{D}\left(x, w^{n}(x)\right) \\
\text { w.r.t. } & x=\left[\alpha, \text { sweep, twist, taper, } x_{C G_{\text {target }}}, C G_{\% M A C}\right] \\
\text { subject to } & R\left(x, w^{n}(x)\right)=0 \\
& C_{L_{\text {target }}}-C_{L}\left(x, w^{n}(x)\right) \leq 0 \\
& 0 \leq C_{m}\left(x, w^{n}(x)\right) \leq 10^{-4} \\
& C_{m_{\alpha}}\left(x, w^{n}(x)\right)+C_{L_{\alpha}}\left(x, w^{n}(x)\right) \times \operatorname{Static} \operatorname{Margin}(x)=0 \\
& -10^{-5} \leq x_{C G}-x_{C G_{\text {target }}} \leq 10^{-5}
\end{aligned}
$$


The last three problems are formulated as multidisciplinary systems. The CG calculation forms one discipline and the aerodynamics forms the second discipline. An interdisciplinary feasible (IDF) approach is used to solve the system. As shown in the problem definitions, the discipline coupling variable is the CG location. A single target variable, $x_{C G_{\text {target }}}$, is added as an input to the aerodynamic discipline and an additional constraint - that $x_{C G}-x_{C G_{\text {target }}}=0$ - is added to the problem to enforce consistency at the optimized solution. In addition, because the CG location and neutral point location are both primarily driven by the sweep variable, the optimizer has been given additional freedom to specify the CG location as a percentage of the distance between the spars $\left(C G_{\% M A C}\right)$. This variable was allowed to vary anywhere in between the spars as well as one MAC length in front of the forward spar. The extra forward freedom is allowed to take into account the potential CG contributions from non-wing portions of the aircraft, such as systems, payload, etc.. Each of these optimizations has been run at Mach=0.8 with a target lift coefficient of 0.3 . The reference temperature, density and pressure are $288.15 \mathrm{~K}, 1.225 \mathrm{~kg} / \mathrm{m}^{3}$ and $101325.0 \mathrm{~Pa}$ respectively. For the static margin constrained optimization, the requested static margin is $5 \%$ of the MAC. Table 4 outlines the limits specified on the various design variables used. The results of the three optimizations are

\begin{tabular}{lrr}
\hline \hline \multicolumn{3}{c}{ Design Variable Bounds } \\
\hline Design Variable & Lower Bound & Upper Bound \\
\hline$\alpha$ (deg.) & -14.32 & 14.32 \\
Sweep (deg.) & -75 & 75 \\
Tip Twist(deg.) & -15 & 15 \\
Taper Ratio & 0.1 & 1.2 \\
$x_{C G_{\text {target }}}(\mathrm{m})$ & -200 & 200 \\
$C G_{\% M A C}$ & -1 & 1 \\
\hline \hline
\end{tabular}

Table 4. Design variable limits

shown in Table 5. Note that in the trim constrained and stability constrained optimizations, the target center of gravity location, $x_{C G_{\mathrm{target}}}$, was used as the reference center for the computation of the moments and their derivatives.

\begin{tabular}{lrrrrr}
\hline \hline \multicolumn{7}{c}{ Optimization Cases } \\
\hline Parameter & ONERA M6 & Lift Constrained & Trimmed & $C_{m_{\alpha}}$ Constrained & Static Margin Constrained \\
\hline$C_{L}$ & 0.30000 & 0.30000 & 0.30000 & 0.30000 & 0.30000 \\
$C_{D}$ & 0.01834 & 0.01438 & 0.01438 & 0.01448 & 0.01479 \\
$C_{m}$ & -0.04897 & -0.04610 & 0.00010 & -0.00001 & -0.00001 \\
$C_{m_{\alpha}}$ & 0.86241 & 0.58871 & 0.15194 & -0.00001 & -0.12271 \\
CG Location(m) & 0.56765 & 1.05927 & 0.96265 & 0.97165 & 0.90127 \\
\hline$\alpha$ (deg.) & 3.65 & 6.88 & 6.91 & 7.38 & 7.74 \\
Sweep (deg.) & 30.0 & 56.96 & 56.96 & 57.79 & 56.00 \\
Tip Twist(deg.) & 0.0 & -6.21 & -6.21 & -7.71 & -9.04 \\
Taper Ratio & 0.562 & 0.304 & 0.304 & 0.268 & 0.298 \\
\hline \hline
\end{tabular}

Table 5. Optimization results comparison

A convergence plot for the five cases shown in Table 5 is shown in Figure 6

\section{Discussion}

As shown in Table 5 all of the optimizations achieved the requested target lift coefficient of 0.3 and have reduced the drag with respect to the baseline case. The lowest drag was achieved in both the lift constrained case and the trim constrained optimizations. The optimizer was able to match the performance of the lift constrained case with the trim constrained case by moving the CG variable to trim the wing instead of altering the aerodynamic shape. As expected, the predicted drag increases with both of the stability constrained 


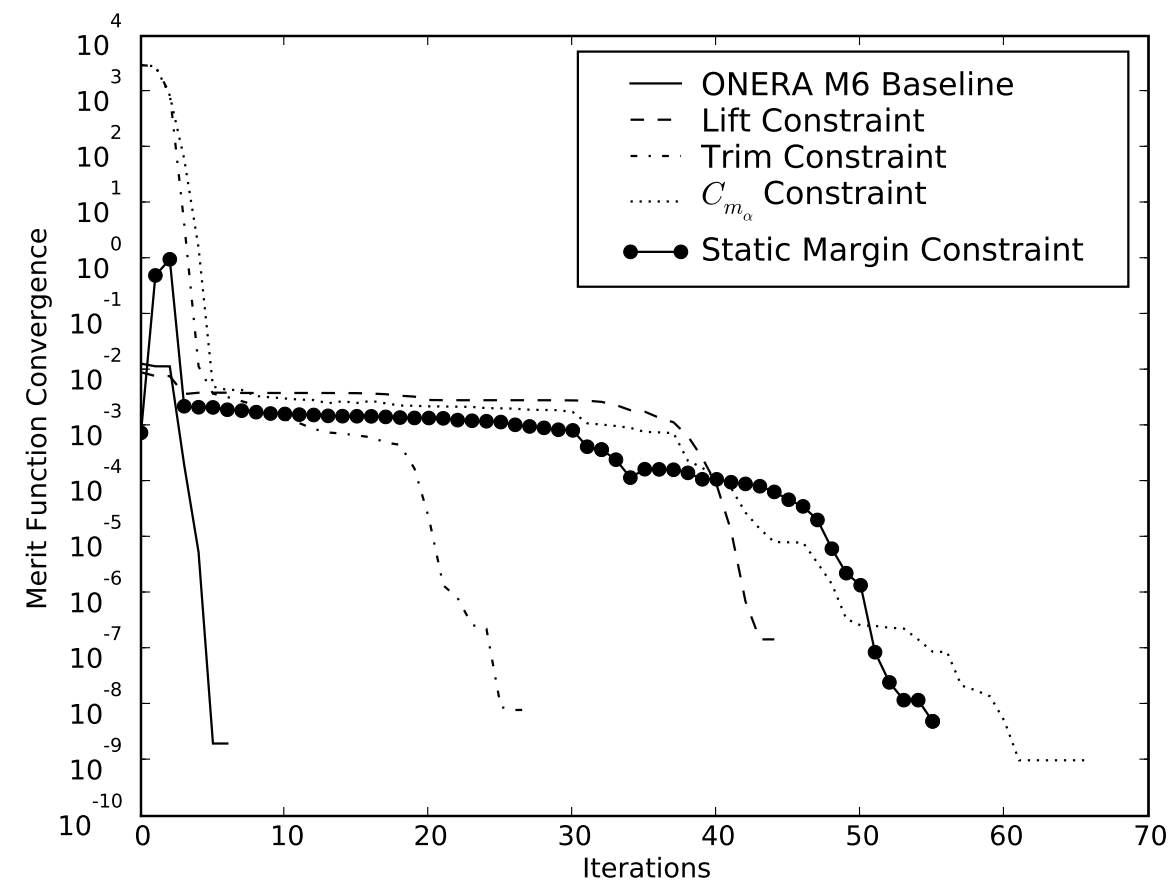

Figure 6. Convergence histories for the various optimization problems

cases. The penalty of the static margin constraint is larger than the $C_{m_{\alpha}}$ constraint because it forces a more negative value of $C_{m_{\alpha}}$ than the default $C_{m_{\alpha}}$ constraint. Looking at the final shape of the design, in all four cases the bulk of the drag minimization comes from the increase in sweep. This change reduces the wave drag caused by the shock on the wing in the baseline case. The trim and stability constraints are satisfied with a combination of changes to the other variables. The most prominent changes appear to be in twist and CG location. Both of the stability constrained cases have more twist than the other two. The static margin case has significantly more twist than any of the others and the CG is significantly farther forward. The resulting flow solutions for each of the cases are plotted in Figures $7(\mathrm{a}) \mid 7(\mathrm{e})$ and a comparison of the resulting lift distributions is shown in Figure 8.

The lift distributions also show what one would expect. In order to counter the typical nose down moment of a symmetric airfoil, the optimizer has shifted lift from the tips inboard. This lift reduction at the tips creates a nose up moment that trims and stabilizes the wing. Note that the baseline case and the trimmed case have the same lift distribution. These two cases have the same aerodynamic shape, but the CG has been moved forward in the trimmed optimization to counteract the aerodynamic moment. Also note that the optimal lift constrained solution is not elliptical. This is primarily because the twist is forced to maintain a linear distribution along the span.

\section{Conclusion}

Stability-constrained optimization is conducted for a flying wing using a time-spectral flow solver and adjoint method. A new time-spectral approach to the computation of stability derivatives is shown to be valid and an ADjoint implementation of the time-spectral adjoint is shown to be computationally efficient and to have accuracy greater than any previous implementation of a time-spectral adjoint. The optimizations conducted with this framework produced sensible and meaningful results. As expected, the optimum stability constrained result has a higher drag coefficient than the baseline lift-constrained case. The trimmed case is similar to the baseline case. The differences evident in the stability constrained case reinforce the fact that for some unconventional designs, such as flying wings, including stability considerations in the design optimization is important. 


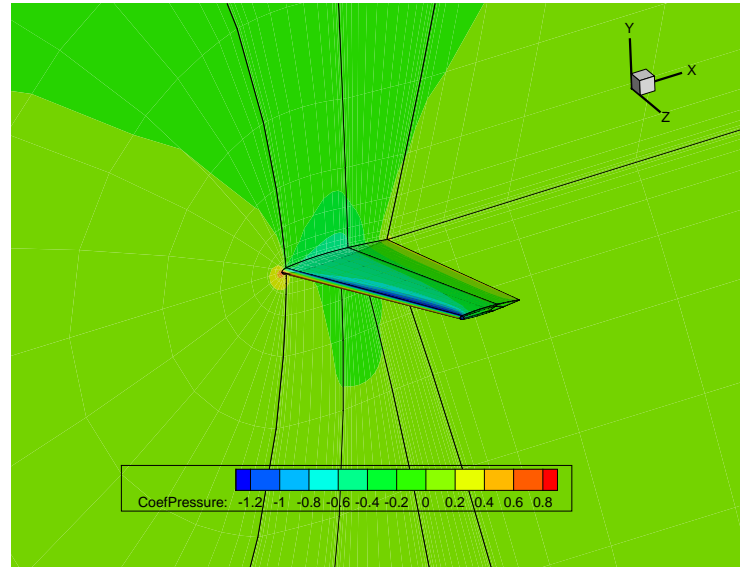

(a) ONERA M6 solution

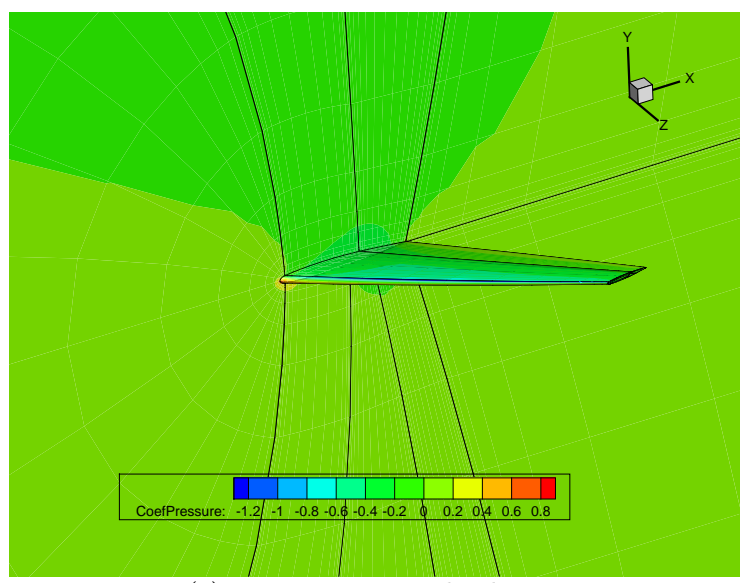

(c) Trim constrained solution

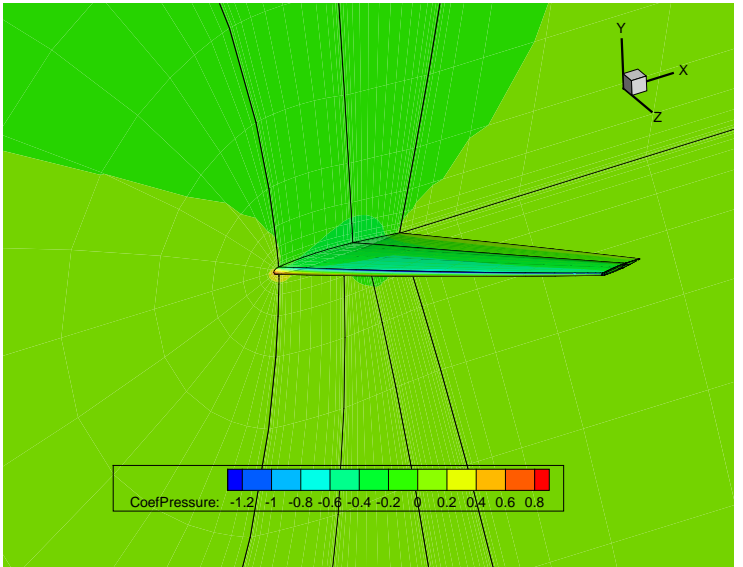

(b) Lift constrained solution

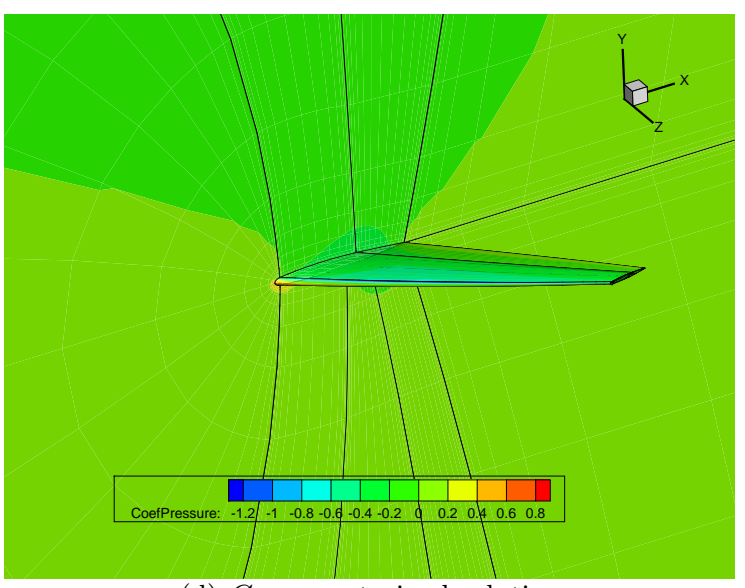

(d) $C_{m_{\alpha}}$ constrained solution

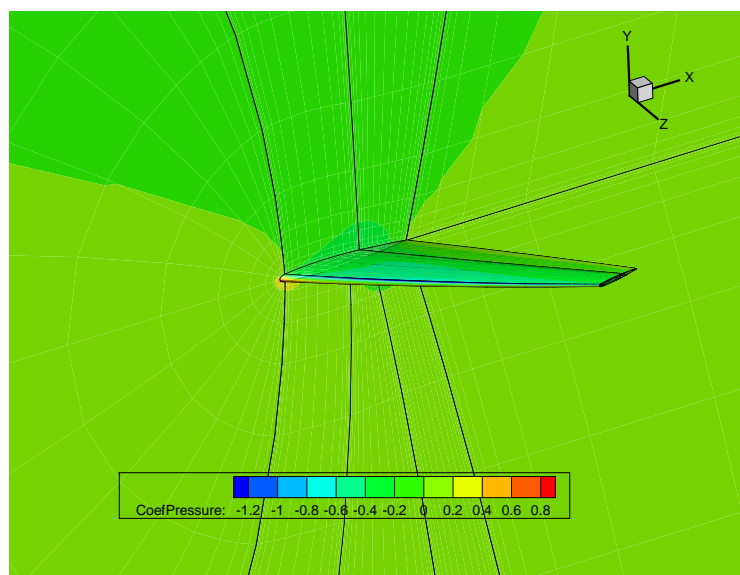

(e) Static margin constrained solution

Figure 7. Optimal Onera M6 results 


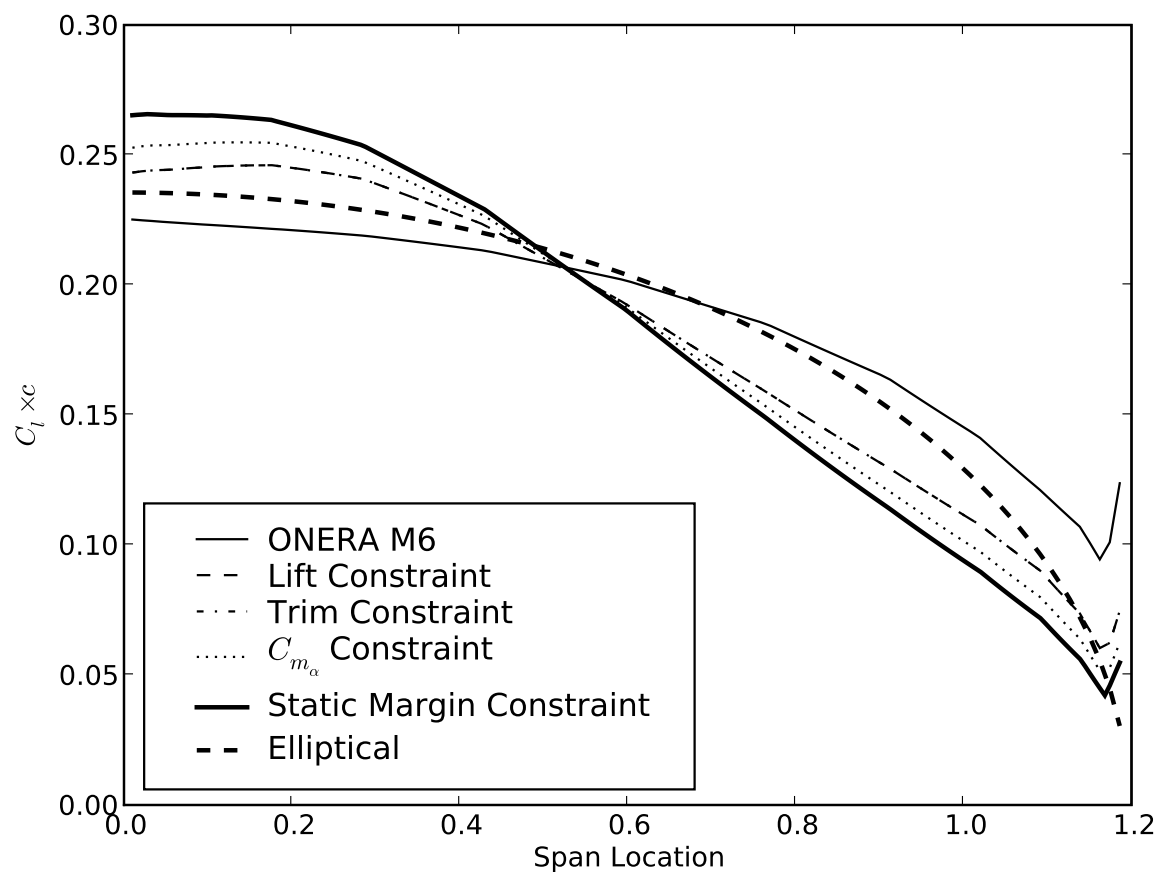

Figure 8. Lift distribution comparison

\section{Acknowledgements}

The authors would like to thank Dr. Edwin van der Weide for his help with the SUmb flow solver. Financial support for this research was provided by the University of Toronto and the Natural Sciences and Engineering Research Council(NSERC) of Canada. Computations were performed on the GPC supercomputer at the SciNet HPC Consortium. SciNet is funded by: the Canada Foundation for Innovation under the auspices of Compute Canada; the Government of Ontario; Ontario Research Fund - Research Excellence; and the University of Toronto. 


\section{References}

${ }^{1}$ Jameson, A., "Aerodynamic Design via Control Theory," Journal of Scientific Computing, Vol. 3, No. 3, sep 1988, pp. 233-260.

${ }^{2}$ Nemec, M. and Zingg, D. W., "Newton-Krylov Algorithm for Aerodynamic Design Using the Navier-Stokes Equations," AIAA Journal, Vol. 40, No. 6, June 2002, pp. 1146-1154.

${ }^{3}$ Hicken, J. and Zingg, D. W., "Aerodynamic Optimization Algorithm with Integrated Geometry Parameterization and Mesh Movement," AIAA Journal, Vol. 48, No. 2, February 2010, pp. 400-413.

${ }^{4}$ Reuther, J. J., Jameson, A., Alonso, J. J., , Rimlinger, M. J., and Saunders, D., "Constrained Multipoint Aerodynamic Shape Optimization Using an Adjoint Formulation and Parallel Computers, Part 1," Journal of Aircraft, Vol. 36, No. 1, 1999, pp. 51-60.

${ }^{5}$ Reuther, J. J., Jameson, A., Alonso, J. J., , Rimlinger, M. J., and Saunders, D., "Constrained Multipoint Aerodynamic Shape Optimization Using an Adjoint Formulation and Parallel Computers, Part 2," Journal of Aircraft, Vol. 36, No. 1, 1999, pp. 61-74.

${ }^{6}$ Gill, P. E., Murray, W., and Saunders, M. A., "SNOPT: An SQP Algorithm for Large-Scale Constrained Optimization," SIAM Review, Vol. 47, No. 1, 2005, pp. 99-131.

${ }^{7}$ Mader, C. A., Martins, J. R. R. A., Alonso, J. J., and van der Weide, E., "ADjoint: An Approach for the Rapid Development of Discrete Adjoint Solvers," AIAA Journal, Vol. 46, No. 4, 2008, pp. 863-873.

${ }^{8}$ van der Weide, E., Gopinath, A. K., and Jameson, A., "Turbomachinery Applications with the Time Spectral Method," AIAA Paper 2005-4905, 2005.

${ }^{9}$ Gopinath, A. K. and Jameson, A., "Time Spectral Method for Periodic Unsteady Computations over Two- Three Dimensional Bodies," AIAA Paper 2005-1220, 2005.

${ }^{10}$ van der Weide, E., Kalitzin, G., Schluter, J., and Alonso, J. J., "Unsteady Turbomachinery Computations Using Massively Parallel Platforms," Proceedings of the 44th AIAA Aerospace Sciences Meeting and Exhibit, Reno, NV, 2006, AIAA $2006-0421$.

${ }^{11}$ Babcock, D. A. and Jr., D. A. S. A., "Estimating Aircraft Stability Derivatives Through Finite Element Analysis," AIAA Paper 2004-5174, Aug. 2004.

${ }^{12}$ Park, M. A., Green, L. L., Montgomery, R. C., and Raney, D. L., "Determination of Stability and Control Derivatives Using Computational Fluid Dynamics and Automatic Differentiation," AIAA Paper 1999-3136, 1999.

${ }^{13}$ Park, M. A. and Green, L. L., "Steady-State Computation of Constant Rotational Rate Dynamic Stability Derivatives," AIAA Paper 2000-4321, 2000.

${ }^{14}$ Limache, A. and Cliff, E., "Aerodynamic Sensitivity Theory for Rotary Stability Derivatives," Journal of Aircraft, Vol. 37 , 2000, pp. $676-683$.

${ }^{15}$ Murman, S. M., "Reduced-Frequency Approach for Calculating Dynamic Derivatives," AIAA Journal, Vol. 45, No. 6, 2007, pp. 1161-1168.

${ }^{16}$ Etkin, B., Dynamics of Atmospheric Flight, Dover Publications, Mineola, New York, 2000.

${ }^{17}$ Pascual, V. and Hascoët, L., "Extension of TAPENADE Towards Fortran 95," Automatic Differentiation: Applications, Theory, and Tools, edited by H. M. Bücker, G. Corliss, P. Hovland, U. Naumann, and B. Norris, Lecture Notes in Computational Science and Engineering, Springer, 2005.

${ }^{18}$ Hascoët, L. and Pascual, V., "TAPENADE 2.1 User's Guide," Technical report 300, INRIA, 2004.

${ }^{19} 2001$.

${ }^{20}$ Balay, S., Buschelman, K., Eijkhout, V., Gropp, W. D., Kaushik, D., Knepley, M. G., McInnes, L. C., Smith, B. F., and Zhang, H., "PETSc Users Manual," Tech. Rep. ANL-95/11 - Revision 2.1.5, Argonne National Laboratory, 2004.

${ }^{21}$ Chai, S., Crisafulli, P., and W.H.Mason, "Aircraft Center of Gravity Estimation in Conceptual/Preliminary Design," Proceedings of the 1st AIAA Aircraft Engineering, Technology, and Operations Congress, Los Angeles, CA, 1995, AIAA 953882.

${ }^{22}$ ESDU, "Geometrical Properties of Cranked and Straight Tapered Wing Planforms," Tech. Rep. Item No. 76003, ESDU International plc, London, UK, January 1976. 